\title{
Rasgos de personalidad, necesidad de cognición y satisfacción vital en estudiantes universitarios chilenos*
}

\author{
Personality Traits, Need for Cognition and Life \\ Satisfaction in Chilean University Students
}

Recibido: noviembre 18 de 2011 | Revisado: diciembre 20 de 2012 | Aceptado: marzo 28 de 2013

\author{
Marcos A. Carmona-Halty ** \\ PAUlina P. ROJAS-PAZ \\ Universidad de Tarapacá, Chile
}

doi:10.11144/Javeriana.UPSY13-1.rpnc

Para citar este artículo: Carmona-Halty, M. A. \& Rojas-Paz, P. P. (2014). Rasgos de personalidad, necesidad de cognición y satisfacción vital en estudiantes universitarios chilenos. Universitas Psychologica, 13(1), 83-93. doi:10.11144/Javeriana. UPSY13-1.rpnc

* La presente investigación contó con el apoyo de la Dirección General de Investigación de la Universidad de Tarapacá y del Convenio de Desemepeño MINEDUC-UTA.

*** La correspondencia relativa a este artículo debe ser dirigida a Marcos Carmona- Halty, Universidad de Tarapacá, Departamento de Ciencias Sociales, Avda. Luis Emilio Recabarren \# 2477, Iquique, Chile.E-mail: mcarmonah@uta.cl

\section{RESUMEN}

El estudio examina el papel de los rasgos de personalidad y la necesidad de cognición en la predicción de la satisfacción vital. Para ello, se evaluó a una muestra de 235 estudiantes de una universidad estatal chilena, utilizando el Inventario Cinco Factores (ICF-UTA), la Escala de Necesidad de Cognición (ENC) y la Escala de Satisfaccion con la Vida (SWLS). Los resultados indican relaciones significativas entre satisfacción y los rasgos de neuroticismo $(-0.4)$, responsabilidad (0.29), afabilidad (0.34) y el constructo de necesidad de cognición (0.32), los que en conjunto explican un porcentaje significativo de varianza de la satisfacción vital de los estudiantes.

Palabras clave autores

Satisfacción vital, personalidad, necesidad de cognición.

Palabras clave descriptores

Pruebas psicológicas, investigación cuantitativa, Chile.

\section{A B S T R AC T}

The study examines the role of personality traits and the need for cognition in predicting life satisfaction. We evaluated a sample of 235 students at a state university in Chile, using the Five-Factor Inventory (ICF-UTA), the Need for Cognition Scale (NCS) and the Scale of Satisfaction with Life (SWLS). Results suggest significant relationships between life satisfaction, and neuroticism (-0.4), conscientiousness (0.29), agreeableness (0.34) and the construct of need for cognition (0.32), which together explain a significant percentage of variance in life satisfaction of students.

Key words authors

Life satisfaction, personality, need for cognition.

Keywords plus

Psychological Test, Quantitative Research, Chile. 


\section{Introducción}

Tradicionalmente, denominamos "felicidad" al bienestar subjetivo experimentado cuando se sienten con mayor frecuencia emociones agradables que desagradables, en ocasiones en que nos encontramos comprometidos en actividades interesantes y estamos satisfechos con nuestra vida (Cuadra \& Florenzano, 2003). El constructo de bienestar subjetivo ha sido abordado desde dos perspectivas diferentes: emocional y cognitiva. En la primera, se considera el equilibrio entre los afectos positivos y negativos experimentados por una persona dentro de un rango determinado de tiempo. En la segunda, se atiende a la valoración que la persona hace de su propia vida, ya sea de manera global o específica, concepto denominado satisfacción vital (Díaz \& Sánchez-López, 2001).

Inicialmente, se investigaron características sociodemográficas en relación con la satisfacción vital de las personas, tales como edad, sexo y nivel de ingresos, las cuales no fueron concluyentes al momento de predecir qué personas estarán satisfechas con su vida y cuáles no (Myers \& Diener, 1995). Posteriormente, y fundamentado en el amplio porcentaje de varianza que explican algunas unidades disposicionales (Castro \& Sánchez-López, 2000), el interés se centró en el estudio de este tipo variables. $\mathrm{Al}$ asociar el bienestar subjetivo a variables disposicionales, es posible asumir características vinculadas a ellas, tales como su estabilidad a través del tiempo y una limitada variación en relación con los eventos vitales (Diener, Oishi \& Lucas, 2003). Según este planteamiento, las personas "felices por naturaleza" son aquellas que experimentan naturalmente mayor satisfacción en los diferentes aspectos de su vida (Castro \& Sánchez-López, 2000). Dentro de las variables disposicionales estudiadas, resulta un hecho constatado empíricamente que los rasgos de personalidad están estrechamente relacionados con el bienestar subjetivo, tanto desde una perspectiva emocional como cognitiva (McCrae \& Costa, 1997), siendo documentados estos hallazgos principalmente a través del modelo de los cinco factores de personalidad. En esta dirección, un metaanálisis efectuado por DeNeve y Cooper (1998) indica que en investigaciones pasadas se utilizaron 137 constructos diferentes de personalidad en relación con la satisfacción vital, siendo el modelo de los cinco factores, el más prometedor en esta área, lo cual ha contribuido a considerar los rasgos de personalidad como el mayor determinante del bienestar (Diener et al., 2003).

De acuerdo a la descripción de Judge, Bono, Ilies y Gerhardt (2002), los cinco factores de la personalidad se caracterizan de la siguiente manera: neuroticismo: refleja la tendencia a experimentar con facilidad emociones displacenteras, tales como ira, ansiedad, depresión y/o vulnerabilidad; extroversión: alude a la propensión a ser enérgico, asertivo, activo, sociable y con tendencia a experimentar afectos positivos; responsabilidad: indica preferencia a trabajar con autodisciplina, mantención del esfuerzo y orientado al logro; afabilidad: revela la orientación a ser compasivo, cooperador, gentil y confiable; y apertura a la experiencia: vinculada al gusto por lo no convencional, creativo, informado y autónomo.

Las investigaciones han reflejado que las dimensiones de neuroticismo y extroversión están relacionadas con la experiencia de afecto negativo y positivo, respectivamente. Además, el balance cognitivo que hace la persona entre estos afectos, "dispuesto" por estos dos grandes rasgos de personalidad, contribuye a la experiencia de la satisfacción vital (Díaz \& Sánchez-López, 2002). En esta dirección, trabajos metaanalíticos han encontrado que el predictor más potente sobre la satisfacción vital, es el factor de neuroticismo (relacionado inversamente); mientras que la relación directa más importante con este constructo fluctúa, según los estudios, entre los factores de extroversión y responsabilidad (Steel, Schmidt \& Schultz, 2008). Además, los datos sugieren que la relación más débil entre personalidad y satisfacción, desde el modelo de los cinco factores, corresponde a la dimensión de apertura a la experiencia (Acevedo, 2010).

En Chile, las investigaciones referidas al ámbito del bienestar son aún escasas y no se corresponden con el actual avance empírico mundial que esta temática posee. Es posible encontrar algunos trabajos publicados en revistas científicas que la proponen 
como un área emergente de estudio (Cuadra \& Florenzano, 2003), en relación con indicadores de salud y apoyo social en adultos mayores (Mella et al., 2004) y variables sociodemográficas (Moyano \& Ramos, 2007), como consecuente del optimismo (Vera-Villarroel, Pávez \& Silva, 2012) y protector de la ansiedad (Pavez, Mena \& Vera-Villarroel, 2012), explorando sus niveles en pueblos originarios del sur del país (Schnettler et al., 2012) y en trabajadores informales (Moyano, Castillo \& Lizana, 2008). Además, recientemente se realizó una compilación que aglutina estudios concernientes a la calidad de vida en general (Moyano, 2010) y la adaptación a población chilena de diferentes escalas de bienestar y felicidad (Horwitz, Bascuñan, Schiattino, Acuña \& Jiménez, 2010; Vera-Villarroel, Celis-Atenas \& Cordova-Rubio, 2011; Vera-Villarroel, Urzúa, Pavez, Celis-Atenas \& Silva, 2012). A pesar del innegable aporte al conocimiento que estos trabajos ofrecen en la materia, a la fecha no se han publicado, que se sepa, trabajos que vinculen rasgos de personalidad y bienestar subjetivo en población chilena, lo cual limita las posibilidades de generalizar las relaciones reportadas por la literatura anglosajona y las potenciales implicancias prácticas que de ellas se derivan. Más aún, cuando los hallazgos sugieren que los predictores de bienestar pueden variar según los diferentes contextos culturales y que no deben tomarse los hallazgos obtenidos en población norteamericana como universales (Castro, 2011).

Por otra parte, abordar esta temática en el contexto universitario parece de especial relevancia debido a que en los últimos años se ha experimentado un aumento en el porcentaje de jóvenes que ingresan a las universidades de Iberoamerica, ya sean públicas o privadas (para una revisión en profundidad, véase Brunner \& Ferrada, 2011). Por ejemplo, en el caso de Chile se observa un incremento de 249.482 estudiantes en 1990 a 1.127 .200 en el año 2010, según cifras del Ministerio de Educación del Gobierno de Chile (www.divesup.cl). Sin embargo, la ampliación de la cobertura educacional no se corresponde con un incremento en el rendimiento académico de los alumnos y, a poco andar, es posible observar que los hábitos de estudio adquiridos en los escalones más bajos del sistema educativo no siempre ayudan a la adaptación natural del estudiante al ámbito universitario (Carloto $\&$ Goncalves, 2008), lo que en última instancia se traduce en un aumento de los índices de mortalidad académica, tal y como reportan cifras del Instituto Nacional de Estadísticas de Chile (www.ine.cl), en donde la mitad de los estudiantes que ingresan a alguna carrera universitaria no están en ella al quinto año, lo cual representa tanto un impacto económico para el sistema como para las familias y el propio estudiante, ya que se ven truncadas sus expectativas de crecimiento y desarrollo académico.

Atendiendo a la actual realidad de la educación superior, es relevante incorporar al estudio de la satisfacción de los universitarios, variables relacionadas con la forma en que estos analizan, recuerdan y emplean la información recibida, para hacer frente a las demandas y exigencias del ámbito académico. Esta inclusión se fundamenta en investigaciones que han demostrado que el acto de estudiar, por sí mismo, promueve la satisfacción de los estudiantes universitarios (Cheung, 2000), y para lo cual resulta esencial comprender y procesar la información de manera efectiva. En esta dirección, Coutinho y Woolery (2004), encontraron relaciones importantes entre los niveles de satisfacción vital y la preferencia por ocuparnos y disfrutar de actividad cognitiva, constructo denominado como necesidad de cognición, logrando explicar un 38\% de la varianza de la satisfacción vital en una muestra de estudiantes norteamericanos. Las personas con altos índices en esta variable, se caracterizan por estar motivadas intrínsicamente para involucrarse en actividad reflexiva, exhibiendo una fuerte tendencia hacia tareas cognitivas complejas. Por el contrario, aquellos que poseen bajos índices en necesidad de cognición son descritos como "avaros cognitivos" los cuales deben ser motivados externamente para gastar su energía en actividad cognitiva (Coutinho \& Woolery, 2004).

Inicialmente, la necesidad de cognición se investigó con la finalidad de evaluar en qué medida afectaba al tipo de estrategia o ruta (central o periférica) que sigue una persona para procesar la información y cambiar sus actitudes. Actualmente, este constructo ha sobrepasado el ámbito de 
estudio del cambio actitudinal, existiendo trabajos que lo ligan con diversas áreas de la psicología. Por ejemplo, se ha encontrado que la necesidad de cognición se relaciona positivamente con la tendencia a desear la máxima información posible (Sorrentino, Bobocel, Guitta, Olson \& Hewitt, 1988), con el grado de influencia interpersonal que poseen los sujetos (Briñol et al., 2005), con la tendencia a prestar atención únicamente a la tarea realizada (Osberg, 1987) y con la preferencia por respaldar los juicios en información empírica (Leary, Sheppard, McNeil, Jenkis \& Barnes, 1986). Por otra parte, se ha encontrado que la necesidad de cognición se relaciona negativamente con el dogmatismo (Webster \& Kruglanski, 1994) y con la tendencia a valorar el atractivo o popularidad como factores importantes de la propia identidad (Berzonsky \& Sullivan, 1992). En un contexto universitario, se ha encontrado que la necesidad de cognición se vincula con un adecuado funcionamiento académico y avance curricular de los alumnos (Leone \& Dalton, 1988), lo cual forma parte de los proyectos y metas personales que los estudiantes tradicionalmente se proponen (Castro, 1998). Además, existe evidencia de que los alumnos con una alta necesidad de cognición son eficaces al momento de procesar la información que requiere mayor esfuerzo cognitivo, lo cual favorecería la obtención de mejores calificaciones (Sadowski \& Gülgöz, 1996).

Según los antecedentes planteados, el presente estudio tiene por objetivo examinar la relación entre personalidad y satisfacción vital propuesta en la literatura e incorporar el constructo de necesidad de cognición como una variable relevante en la comprensión de la satisfacción vital de los estudiantes universitarios. Además, se examinará el papel predictivo de dichas variables en la satisfacción vital de este segmento de la población. Específicamente, las hipótesis de trabajo son las siguientes: (a) se encontrará una relación significativa e inversa entre el factor de neuroticismo y los niveles de satisfacción vital; (b) se encontrará una relación significativa entre el factor de extroversión y los niveles de satisfacción vital; (c) se encontrará una relación significativa entre el factor de responsabilidad y los niveles de satisfacción vital; (d) se encontrará una relación significativa entre el constructo de necesidad de cognición y los niveles de satisfacción vital y (e); las variables hipotetizadas, en su conjunto, predecirán parcialmente la varianza del constructo de satisfacción vital.

\section{Método}

\section{Participantes}

La muestra está compuesta por 235 estudiantes de primer y segundo año de una universidad estatal chilena, correspondientes a las carreras de psicología, trabajo social, ingeniería comercial, kinesiología y enfermería, de los cuales 80 (34.4\%) son hombres y 155 (65.6\%) mujeres con una edad media de 21 años $(D E=1.8)$.

\section{Instrumentos de evaluación}

\section{Inventario Cinco Factores UTA (ICF-UTA)}

Se utilizó la adaptación chilena del Modelo de Personalidad de Cinco Factores desarrollada por Cuadra, Caballero, Gilardi y Miño (2004). El instrumento consta de 110 reactivos (96 referidos a las cinco dimensiones de la personalidad y 14 a una escala de deseabilidad social) evaluados con una escala de tipo Likert con puntuaciones comprendidas entre 1 (desacuerdo), 2 (a veces) y 3 (de acuerdo), con índices de confiabilidad aceptables en cada una de sus dimensiones (neuroticismo: 0.86; extroversión: 0.83; responsabilidad: 0.83 ; afabilidad: 0.75 y apertura a la experiencia 0.81 ) (Cuadra, Ferrer \& Carmona-Halty, 2007). Además, la validez convergente fue evaluada con la versión en español del inventario 16PF-5 (Russel \& Karol, 1995), encontrando relaciones medias y altas entre las dimensiones de extroversión, animación $(\mathrm{F}+)$ $(r=0.51)$ y atrevimiento $(\mathrm{H}+)(r=0.4)$; neuroticismo, estabilidad $(\mathrm{C}+)(r=-0.68)$, vigilancia $(\mathrm{L}+)$ $(r=0.39)$ y tensión $\left(\mathrm{Q}_{4}+\right)(r=0.42)$; responsabilidad, perfeccionismo $\left(\mathrm{Q}_{3+}\right)(r=0.55)$ y atención a las normas $(\mathrm{G}+)(r=0.47)$; afabilidad, afabilidad $(\mathrm{A}+)(r=0.64)$ y dominancia $(\mathrm{E}-)(r=0.55)$; apertura a la experiencia y apertura al cambio 
$\left(\mathrm{Q}_{1}+\right)(r=0.67) ; \mathrm{MMPI}$, con relaciones entre neuroticismo y psicastenia $(r=0.57)$ y extroversión e introversión $(r=0.58)$ y EPQ, con relaciones entre escalas del mismo nombre, extroversión $(r=0.72)$ y neuroticismo $(r=0.83)$ (Cuadra et al., 2004).

\section{Escala de Satisfacción con la Vida (SWLS)}

Se utilizó la versión en español de la Escala de Satisfacción con la Vida (Diener, Emmons, Larsen \& Griffin, 1985), adaptada por Atienza, Pons, Balaguer y García-Merita (2000). Dicho instrumento consta de 5 ítems (p. e., "las condiciones de mi vida son excelentes") evaluados con una escala de tipo Likert con puntuaciones comprendidas entre 1 (totalmente en desacuerdo) y 7 (totalmente de acuerdo), con una solución monofactorial que llega a explicar el 53\% de la varianza y una alta consistencia interna con un índice alfa de 0.84. En población chilena, es posible encontrar indicadores similares de fiabilidad, que oscilan entre 0.82 (Cuadra et al., 2007) y 0.87 (Moyano \& Ramos, 2007).

\section{Escala de Necesidad de Cognición (ENC)}

Se utilizó la versión reducida de la Escala de Necesidad de Cognición (Cacioppo \& Petty, 1982), adaptada al español por Falces, Briñol, Sierra, Becerra y Alier (2001) y estandarizada para población chilena por Simonetti, Haberland, Bohner y González (2007), la que cuenta con un índice de consistencia interna aceptable de 0.85 . El instrumento consta de 18 ítems (p. e., "me atrae la idea de tener que pensar mucho para conseguir algo") evaluados mediante una escala de tipo Likert con puntuaciones comprendidas entre 1 (nada característico de mí) y 7 (muy característico de mí), con un índice de confiabilidad de 0.81 (Falces et al., 2001). Su estructura factorial indica la presencia de un único factor que explica alrededor de un 30\% de la varianza (Cacioppo, Petty \& Kao, 1984).

\section{Procedimientos}

Los estudiantes accedieron voluntariamente a la investigación, siendo contactados en sus respectivos horarios de clase y formalizando su participación a través de un consentimiento escrito. A continuación se les explicaron los objetivos y aspectos generales del estudio, siguiendo las pautas éticas internacionales para la investigación biomédica con seres humanos del Council for International Organizations of Medical Sciences (2002). Posteriormente, contestaron los instrumentos de manera grupal y anónima, siguiendo un procedimiento de lápiz y papel con un tiempo promedio de respuesta de 40 minutos, aproximadamente.

\section{Resultados}

Los estadísticos descriptivos, índices de consistencia interna y matriz de correlación de las variables incluidas en el estudio se muestran en la Tabla 1. En todas las dimensiones se observan indicadores aceptables de confiabilidad, con valores comprendidos entre 0.73 y 0.82 , los cuales resultan similares a los obtenidos en estudios anteriores (Cuadra, Carmona-Halty \& Cabrera, 2008). Para examinar la relación entre personalidad, necesidad de cognición y satisfacción vital, se realizó un análisis de correlación de Pearson entre los puntajes obtenidos en la Escala de Satisfacción Vital, las dimensiones del Inventario Cinco Factores - UTA y la Escala de Necesidad de Cognición. Los resultados muestran que los factores de neuroticismo, $r(235)=-0.4$, $p=0.01$; responsabilidad, $r(235)=0.29, p=0.01$; afabilidad, $r(235)=0.34, p=0.01$ y el constructo de necesidad de cognición, $r(235)=0.32, p=0.01$ obtienen una relación media con la satisfacción vital (inversa en el caso de neuroticismo), según el estándar propuesto por Cohen (1992); mientras que los factores de extroversión, $r(235)=0.17, p=0.01$ y apertura a la experiencia, $r(235)=0.23, p=0.01$ obtienen relaciones pequeñas.

Para examinar el papel de los rasgos de personalidad y la necesidad de cognición en la predicción de la satisfacción vital, se realizó un análisis de regresión lineal por pasos sucesivos (step-wise) (Tabla 2), incluyendo el sexo y la edad de los participantes como variables sociodemográficas. El modelo predictor comprende las dimensiones de neuroticismo, $B=-0.28, p=0.01,95 \%$ IC $[-0.3$, 
TABLA 1

Promedio, desviación estándar, confiabilidad y matriz de correlación de las variables estudiadas

\begin{tabular}{lccccccccc}
\hline & $M$ & $D E$ & 1 & 2 & 3 & 4 & 5 & 6 & 7 \\
\hline 1. Satisfacción vital & 25.51 & 5.42 & 0.79 & & & & & & \\
2. Neuroticismo & 41.73 & 7.25 & $0.4^{*}$ & 0.82 & & & & & \\
3. Responsabilidad & 26.57 & 5.37 & $0.29^{*}$ & -0.17 & 0.81 & & & & \\
4. Extroversión & 31.61 & 3.04 & $0.17^{*}$ & -0.13 & 0.08 & 0.8 & & & \\
5. Afabilidad & 24.03 & 4.63 & $0.34^{*}$ & $-0.35^{*}$ & $0.37^{*}$ & $0.29^{*}$ & 0.73 & & \\
6. Apertura a la experiencia & 26.95 & 5.11 & $0.23^{*}$ & $-0.23^{*}$ & $0.3^{*}$ & 0.05 & $0.36^{*}$ & 0.81 & \\
7. Necesidad de cognición & 89.34 & 14.45 & $0.32^{*}$ & $-0.23^{*}$ & $0.29^{*}$ & 0.03 & $0.23^{*}$ & $0.52^{*}$ & 0.79 \\
\hline
\end{tabular}

Nota. En la diagonal se ubica el índice alpha de Cronbach de cada dimensión evaluada.

$* p<0.01$.

Fuente: elaboración propia.

TABLA 2

Modelo de regresión lineal predictor de satisfacción vital

\begin{tabular}{lccc}
\hline & B & Beta & $R$ Parcial \\
\hline Sexo & 0.20 & 0.09 & \\
Edad & 0.18 & 0.01 & \\
Neuroticismo & -0.21 & $-0.28^{*}$ & $-0.28^{*}$ \\
Responsabilidad & 0.13 & $0.13^{*}$ & $0.18^{*}$ \\
Extroversión & 0.12 & 0.07 & 0.07 \\
Afabilidad & 0.16 & $0.14^{*}$ & $0.2^{*}$ \\
Apertura a la experiencia & 0.03 & 0.02 & 0.03 \\
Necesidad de cognición & 0.07 & $0.19^{*}$ & $0.19^{*}$ \\
\hline
\end{tabular}

$* p<0.01$

Fuente: elaboración propia.

-0.12]; responsabilidad, $B=0.13, p=0.04,95 \%$

IC $[0,0.25]$; afabilidad, $\beta=0.14, p=0.04,95 \%$ IC $[0,0.32]$ y necesidad de cognición, $B=0.19, p=$ $0.01,95 \%$ IC $[0.02,0.12]$, explicando en su conjunto un $27 \%$ de la varianza de la satisfacción vital $\left(R^{2}\right.$ $=0.266)$, siendo significativo, $F(6,229)=13.83$, $p=0.01$. Además, se presentan las correlaciones parciales entre cada variable predictora y la variable satisfacción, controlando el efecto para las otras variables predictoras, con la finalidad de complementar la información entregada por el estadístico $ß$ en la ecuación de regresión.

\section{Discusión}

En relación con la primera hipótesis, el factor de neuroticismo es el que alcanza la relación más alta con la satisfacción vital de los estudiantes, hallazgo que se asemeja a lo reportado en trabajos previos (Chico, 2006) y a revisiones que lo proponen como el principal predictor de satisfacción vital (Steel et al., 2008). Respecto de la segunda hipótesis, la relación obtenida por el factor extroversión, si bien resulta ser significativa en la matriz de correlación, en el análisis de regresión, no se reveló como una variable relacionada de forma estadísticamente significativa con la satisfacción vital, lo cual establece una variante respecto de los resultados reportados en la literatura anglosajona. En donde se propone como un determinante central del bienestar subjetivo (Cheng \& Furnham, 2001; DeNeve \& Cooper, 1998). En la tercera hipótesis, el factor responsabilidad obtiene una relación media, tal y como se presenta en trabajos metaanalíticos que lo señalan como uno de los factores de mayor relevancia (Acevedo, 2010). Un resultado interesante 
no hipotetizado, es la relación entre satisfacción y afabilidad (Tabla 1), la cual no aparece referenciada en trabajos anteriores, sugiriendo un matiz local en el que valdría la pena profundizar en futuros trabajos, con la finalidad de corroborar los datos ofrecidos y establecer si se trata de un elemento importante en la población chilena en general, o un fenómeno que surge únicamente en el segmento evaluado. En este sentido, una explicación alternativa a tal resultado podría indicar la dificultad del ICF-UTA para discrminar entre los factores de afabilidad y extroversión, ambos vinculados a las relaciones sociales. Lo cual plantea la necesidad de verificar estos resultados aplicando instrumentos de personalidad que sean utilizados habitualmente en el contexto de investigación.

En cuanto a la cuarta hipótesis, se observa una relación media entre ambos constructos, en la misma dirección de estudios similares (Coutinho \& Woolery, 2004). Resultado coherente con trabajos que examinan la relación satisfacción-personalidad desde el modelo propuesto por Millon (1997), donde se han descrito mayores niveles de satisfacción en personas que poseen rasgos vinculados con un procesamiento de información analítico y toma de decisiones basadas en juicios objetivos (Díaz \& Sánchez-López, 2002), ambas características relacionadas al constructo de necesidad de cognición. Una de las posibles explicaciones que proponemos a la relación reportada, radica en el hecho de que los estudiantes con una alta necesidad de cognición, encontrarían en el procesamiento analítico de la información, una fuente constante de gratificación, en donde la demanda de la tarea estaría asociada con la puesta en práctica de las habilidades cognitivas de los jóvenes, por ende, el acto de estudiar, les reportaría experiencias agradables tal como han demostrado trabajos anteriores (Cheung, 2000). Otra explicación dice relación con la adecuada adaptación al contexto universitario con la que podrían contar los estudiantes con una alta necesidad de cognición, en contraste con aquellos alumnos que no han adquirido adecuados hábitos de estudio en los escalones más bajos del sistema educativo. En este sentido, a los alumnos que disfrutan de la actividad cognitiva les resultaría más sencillo adaptarse a las condiciones de la vida universitaria, ya que las exigencias que se les presentan estarían en sintonía con sus preferencias y actividades que gustan realizar. Por último, tanto disfrutar de las actividades universitarias que implican esfuerzo cognitivo como poseer las herramientas necesarias para adaptarse rápidamente al contexto educacional superior, se traduciría en un exitoso avance curricular y académico, fuente relevante a la hora de construir los juicios sobre satisfacción de los estudiantes, los cuales habitualmente se plantean como proyecto personal: cursar y finalizar sus estudios exitosamente (Castro, 1998).

Un dato vinculado al constructo de necesidad de cognición que nos parece importante señalar es la alta relación encontrada entre necesidad de cognición y apertura a la experiencia (Tabla 1). La cual podría indicar que la necesidad de cognición forma parte de esta dimensión básica de la personalidad. Afirmación que, al menos teóricamente, tiene sentido si aludimos al rasgo especifico de Ideas propuesto (como una de las facetas de apertura a la experiencia) por Costa y McCrae (1999) en la operativización del NEO PI-R. Este rasgo hace referencia a la curiosidad intelectual, en donde aquellos que puntúan alto en la escala Ideas, disfrutan de las discusiones filosóficas y de los rompecabezas mentales, mientras que los que puntúan bajo en esta escala centran sus recursos intelectuales en temas limitados. En este sentido, el hecho de que la investigación previa haya encontrado una baja relación entre apertura a la experiencia y satisfacción vital (corroborada en nuestro estudio) podría deberse a que no se ha analizado la relación especifica de la faceta Ideas, en lugar de la dimensión global de apertura a la experiencia, en una muestra de estudiantes universitarios.

En relación con la quinta hipótesis, el modelo propuesto en esta investigación plantea que los rasgos de neuroticismo, responsabilidad, afabilidad y necesidad de cognición, son los que mayor influencia poseen en la predicción de la satisfacción vital de los estudiantes, logrando explicar un $27 \%$ de la varianza total de esta. En definitiva y según los datos encontrados, los estudiantes que alcanzan los mayores niveles de satisfacción con sus vidas 
son aquellos que poseen una adecuada estabilidad emocional (bajo neuroticismo), interactúan con el resto de manera afable, responden con compromiso y responsabilidad frente a sus obligaciones y disfrutan al vincularse en actividades que implican esfuerzo cognitivo.

Como se señaló anteriormente, la educación superior en Latinoamérica en general y para el caso chileno en particular, se ha expandido a gran escala, acogiendo en la actualidad a jóvenes que en años anteriores hubiesen quedado marginados del sistema. Este fenómeno plantea a las universidades el desafío de ofrecer una educación de calidad que considere todas las variables implicadas en el proceso de enseñanza-aprendizaje, para lo cual resulta importante la identificación de facilitadores y obstaculizadores tanto de adaptación como de permanencia de los alumnos. En este sentido, los datos ofrecidos nos parecen relevantes ya que podrían orientar a las instituciones educativas a considerar la promoción de este tipo de variables, las que lograrían operar como facilitadores de una mejor adaptación a las actividades y exigencias del ámbito universitario. Sin embargo, se debe ser cuidadoso con esta idea ya que actualmente no se cuenta con un estudio empírico que respalde lo dicho por lo que resultaría necesario abordar en futuras investigaciones la inclusión de estas características como variables predictoras, por ejemplo, del rendimiento académico, avance curricular o deserción de los estudiantes. Otro elemento a considerar dentro de las instituciones universitarias, y que podría influir en un incremento de la satisfacción de los estudiantes, dice relación con la mejora de los sistemas de orientación vocacional, los que podrían promover que los jóvenes opten por opciones profesionales que tengan mayores probabilidades de otorgarles satisfacción. En este sentido, nos parece que una mejora en los sistemas de comunicación, publicidad e información precisa de las habilidades necesarias para adaptarse y cumplir con los requisitos de cada institución o malla curricular, debiera hacer más probable un adecuado ajuste entre las características del estudiante y la carrera a la cual opte, ya sea técnica o universitaria.
Las limitaciones del presente estudio radican en el hecho de que, al ser un estudio correlacional, podrían existir terceras variables que expliquen las relaciones encontradas entre los constructos aquí evaluados. Dicha limitación se intenta reducir parcialmente al incluir en el análisis de regresión, las variables de sexo y edad, pero que sin duda no agotan las posibilidades de otras variables que pudieran influir. Además, el carácter transversal del estudio, no permite establecer cual es la dirección de las relaciones entre las dimensiones básicas de la personalidad o necesidad de cognición y la satisfacción vital de los estudiantes universitarios chilenos.

Investigaciones futuras podrían desarrollar trabajos que permitan avanzar en la literatura relacionada con variables cognitivas y satisfacción. Parece necesario indagar en estudios que efectivamente evalúen si los jóvenes universitarios con alta necesidad de cognición derivan parte de su satisfacción vital desde la preferencia por abordar tareas cognitivas complejas. Para ello habría que comparar, por ejemplo, una muestra de estudiantes universitarios con una muestra similar de personas que no estudien en la universidad, en donde la necesidad de cognición debiese ser un predictor significativo únicamente del primer grupo. Otra posibilidad seria evaluar el rol de la necesidad de cognición en la relación entre la evaluación que hacen las personas respecto de distintas áreas de satisfacción (p. e., familia, trabajo, amistades). En donde cabría esperar que las personas con una alta necesidad de cognición no exhiban mayor relación entre las distintas áreas vitales (la evaluación positiva o negativa en un área determinada no afectaría las otras), derivando su satisfacción de elementos específicos del ambiente (procesamiento de información botton-up o abajo-arriba). Esto, en contraste con las personas con una baja necesidad de cognición, quienes podrían manifestar mayor relación entre los juicios que realizan de las distintas áreas vitales (la evaluación positiva o negativa en un área determinada afectaría la evaluación en las otras), derivando su satisfacción de inferencias heurísticas como plantea la hipótesis del estado de ánimo como información (Rodríguez \& Betancor, 2007). En donde el sujeto 
utiliza su afectividad como fuente de información que influencia directamente la toma de decisión y el juicio (Páez \& Carbonero, 1993).

Finalmente, nos parece importante indagar en el porcentaje de varianza que las variables contenidas en este estudio no logra explicar. En esta dirección, se podrían incluir modelos alternativos de personalidad al momento de predecir el bienestar, como el propuesto por Millon (1997), el cual ha recibido un creciente apoyo por parte de los investigadores en esta área (Castro, 2000; Díaz \& Sánchez-López, 2002). Además de la incorporación de variables vinculadas al funcionamiento óptimo de la personalidad tales como el modelo multidimensional de bienestar psicológico (Ryff, 1989) y los constructos de personalidad resistente (Kobasa, 1979) o engagement (Salanova, Schaufeli, Llorens, Peiró \& Grau, 2000), que eventualmente podrían aumentar la predicción del constructo abordado en este estudio.

\section{Referencias}

Acevedo, C. (2010). An investigation of the relationship between Big Five and narrow personality traits and life satisfaction in college student and adult samples. Tesis doctoral no publicada, University of Tennessee, Tennessee, Estados Unidos.

Atienza, F., Pons, D., Balaguer, I. \& García-Merita, M. (2000). Propiedades psicométricas de la Escala de Satisfacción con la Vida en adolescentes. Psicothema, 12(2), 314-319.

Berzonsky, M. \& Sullivan, C. (1992). Social-cognitive aspects of identity stye: Need for cognition, experiential openness and introspection. Journal of Adolescent Research, 7(2), 140-155.

Briñol, P., Becerra, A., Díaz, D., Valle, C., Horcajo, J. \& Gallardo, I. (2005). El efecto de la necesidad de cognición sobre la influencia interpersonal. Psicothema, 17(4), 645-650.

Brunner, J. J. \& Ferrada, R. (2011). Educación superior en Iberoamerica: informe 2011. Santiago, Chile: Centro Interuniversitario de Desarrollo.

Cacioppo, J. \& Petty, R. (1982). The need for cognition. Journal of Personality and Social Psychology, 42(1), 116-131.
Cacioppo, J., Petty, R. \& Kao, C. (1984). The efficient assessment of need for cognition. Journal of Personality Assessment, 48(3), 306-307.

Carloto, M. \& Goncalves, S. (2008). Preditores da síndrome de burnout em estudantes universitários. Pensamiento Psicológico, 4(10), 101-109.

Castro, A. (1998). Diferencias individuales en la estructuración de los proyectos de vida y la percepción subjetiva del bienestar en estudiantes universitarios españoles. Tesis de licenciatura no publicada, Universidad Complutense de Madrid, Madrid, España.

Castro, A. (2000). Estilos de personalidad, objetivos de vida y satisfacción vital: un estudio comparativo con adolescentes argentinos. Tesis de doctorado no publicada, Universidad Complutense de Madrid, Madrid, España.

Castro, A. (2011). Las rutas de acceso al bienestar. Relaciones entre bienestar hedónico y eudaemónico. Un estudio en población Argentina. Revista Iberoamericana de Diagnóstico y Evaluación Psicologica, 31(1), 37-57.

Castro, A. \& Sánchez-López, M. (2000). Objetivos de vida y satisfacción autopercibida en estudiantes universitarios. Psicothema, 12(1), 87-92.

Cohen, J. (1992). A power primer. Psychological Bulletin, 112(1), 155-159.

Cheng, H. \& Furnham, A. (2001). Atributional style and personality as predictors of happiness and mental health. Journal of Happiness Studies, 2(3), 307-327.

Cheung, C. (2000). Studying as a source of life satisfaction among university students. College Student Journal, 34(1), 79-96.

Chico, E. (2006). Personality dimensions and subjective well-being. The Spanish Journal of Psychology, 9(1), 38-44.

Council for International Organizations of Medical Sciences. (2002). Pautas éticas internacionales para la investigación biomédica en seres humanos. Geneva, Suiza: Autor.

Coutinho, S. \& Woolery, L. (2004). The need for cognition and life satisfaction among college students. College Student Journal, 38(2), 203-206.

Costa, P. T. \& McCrae, R. R. (1999). Inventario de personalidad NEO revisado (NEO PI-R) e inventario NEO reducido de cinco factores (NEO-FFI). Madrid: TEA Ediciones, S.A. 
Cuadra, A., Caballero, D., Gilardi, P. \& Miño, G. (2004). Adaptación y estandarización del Inventario de Cinco Factores (ICF-UTA) basado en la teoría de personalidad de los big five. Tesis de licenciatura no publicada, Universidad de Tarapacá, Arica, Chile.

Cuadra, A., Ferrer, R. \& Carmona-Halty, M. (2007, octubre). Estilos de personalidad y bienestar subjetivo. Ponencia presentada en II Congreso Chileno de Psicología: repensando la Psicología en Chile. Nuevos contextos, nuevos temas para la ciencia y la profesión, Santiago, Chile.

Cuadra, A., Carmona-Halty, M. \& Cabrera, S. (2008). Satisfacción vital y necesidad de cognición. Tesis de licenciatura no publicada, Universidad de Tarapacá, Arica, Chile.

Cuadra, H. \& Florenzano, R. (2003). El bienestar subjetivo: hacia una psicología positiva. Revista de Psicología de la Universidad de Chile, 12(1), 83-96.

DeNeve, K. \& Cooper, H. (1998). The happy personality: A meta-analysis of 137 personality traits and subjective well-being. Psychological Bulletin, 124(2), 197-229.

Díaz, J. \& Sánchez-López, M. (2001). Relevancia de los estilos de personalidad y las metas personales en la predicción de la satisfacción vital. Anales de Psicología, 17(2), 151-158.

Díaz, J. \& Sánchez-López, M. (2002). Relaciones entre estilos de personalidad y satisfacción autopercibida en diferentes áreas vitales. Psicothema, 14(1), 100-105.

Diener, E., Emmons, R., Larsen, R. \& Griffin, S. (1985). The Satisfaction with Life Scale. Journal of Personality Assessment, 49(1), 71-75.

Diener, E., Oishi, S. \& Lucas, R. (2003). Personality, culture, and subjective well-being: Emotional and cognitive evaluations of life. Annual Review of Psychology, 54, 403-425.

Falces, C., Briñol, P., Sierra, B., Becerra, A. \& Alier, E. (2001). Validación de la Escala de Necesidad de Cognición y su aplicación al estudio del cambio de actitudes. Psicothema, 13(4), 622-628.

Horwitz, N., Bascuñan, L., Schiattino, I., Acuña, J. \& Jiménez, J. P. (2010). Validación de una escala para medir bienestar subjetivo de los médicos (BISUMED). Revista Medica de Chile, 138(9), 1084-1090.
Judge, T., Bono, J., Ilies, R. \& Gerhardt, M. (2002). Personality and leadership: A qualitative and quantitative review. Journal of Applied Psychology, 87(4), 765-780.

Kobasa, S. C. (1979). Personality and resistance to illness. American Journal of Community Psychology, 7(4), 413-423.

Leary, M., Sheppard, J., McNeil, M., Jenkis, T. \& Barnes, B. (1986). Objetivism in information utilization: Theory and measurement. Journal of Personality Assessment, 50, 32-43.

Leone, C. \& Dalton, C. (1988). Some effects of the need for cognition on course grades. Perceptual and Motor Skills, 67(1), 175-178.

McCrae, R. \& Costa, P. (1997). Personality trait structure as a human universal. American Psychologist, 52(5), 509-516.

Mella, R., Gonzáles, L., D’Appolonio, J., Maldonado, I., Fuenzalida, A. \& Diaz, A. (2004). Factores asociados al bienestar subjetivo en el adulto mayor. Psykhe, 13(1), 79-89.

Millon, T. (1997). MIPS: Inventario Millon de Estilos de Personalidad. Buenos Aires: Paidós.

Moyano, E. (2010). Calidad de vida y psicología en el bicentenario de Chile. Talca: Universidad de Talca.

Moyano, E. \& Ramos, A. (2007). Bienestar subjetivo: midiendo satisfacción vital, felicidad y salud en población chilena de la región Maule. Revista Universum, 22(2), 1-19.

Moyano, E., Castillo, R. \& Lizana, J. (2008). Trabajo informal: motivos, bienestar subjetivo, salud y felicidad en vendedores ambulantes. Psicologia em Estudo, 13(4), 693-701.

Myers, D. \& Diener, E. (1995). Who is happy? Psychological Science, 6(1), 10-19.

Osberg, T. (1987). The convergent and discriminant validity of the Need for Cognition Scale. Journal of Personal Assessment, 51(3), 441-450.

Páez, D. \& Carbonero, A. (1993). Afectividad, cognición y conducta social. Psicothema, 5(Supl. 1), 133-150.

Pavez, P., Mena, L. \& Vera-Villarroel, P. (2012). El rol de la felicidad y el optimismo como factor protector de la salud. Universitas Psychologica, 11(2), 369-380.

Rodríguez, A. \& Betancor, V. (2007). La cognición social. En F. Morales (Ed.), Psicología social (pp. 124-167). Madrid: McGraw Hill. 
Russel, M. T. \& Karol, D. L. (1995). 16PF-5: cuestionario factorial de personalidad (5a. ed.). Madrid: TEA Ediciones, S.A.

Ryff, C. (1989). Happiness is everything, or is it? Explorations on the meaning of psychological well-being. Journal of Personality and Social Psychology, 57(6), 1069-1081.

Sadowski, C. \& Gülgöz, S. (1996). Elaborative processing mediates the relationship between the need for cognition and academic performance. Journal of Psychology, 130(3), 303-308.

Salanova, M., Schaufeli, W., Llorens, S., Peiró, J. \& Grau, R. (2000). Desde el "burnout" al "engagement": ¿una nueva perspectiva? Revista de Psicología del Trabajo y las Organizaciones, 16(1), 17-134.

Schnettler, B., Miranda, H., Sepulveda, J., Denegri, M., Mora, M. \& Lobos, G. (2012). Satisfaction with life and food-related life in persons of the Mapuche ethnic group in Southern Chile: A comparative analysis using logit and probit models. Journal of Happiness Studies, 13(2), 225-246.

Simonetti, C., Haberland, F., Bohner, G. \& González, R. (2007, octubre). Validación de la Escala de Necesidad de Cognición en el contexto chileno. Ponencia presentada en II Congreso Chileno de Psicología: repensando la Psicología en Chile. Nuevos contextos, nuevos temas para la ciencia y la profesión, Santiago, Chile.

Sorrentino, R., Bobocel, D., Guitta, M., Olson, J. \& Hewitt, E. (1988). Uncertainty orientation and persuasion: Individual differences in the effects of personal relevance of social judgements. Journal of Personality and Social Psychology, 46, 189-206.

Steel, P., Schmidt, J. \& Schultz, J. (2008). Refining the relationship between personality and subjective well-being. Psychological Bulletin, 134(1), 138-161.

Vera-Villarroel, P., Pávez, P. \& Silva, J. (2012). El rol predisponente del optimismo: hacia un modelo etiológico del bienestar. Terapia Psicológica, 30(2), 77-84.

Vera-Villarroel, P., Urzúa, A., Pavez, P., Celis-Atenas, K. \& Silva, J. (2012). Evaluation of subjective wellbeing: Analysis of the Satisfaction with Life Scale in Chilean population. Universitas Psychologica, 11(3), 719-727.

Webster, D. \& Kruglanski, A. (1994). Need for cognition and need for cognitive closure. Journal of Personality and Social Psychology, 67, 1049-1052. 
